\title{
The Influence of Subthalamic Nucleus Lesions on Sign-Tracking to Stimuli Paired with Food and Drug Rewards: Facilitation of Incentive Salience Attribution?
}

\author{
Jason M Uslaner*,', James M Dell'Orco ${ }^{2}$, Alex Pevzner ${ }^{3}$ and Terry E Robinson ${ }^{2}$ \\ 'Department of Schizophrenia, Merck Research Laboratories, West Point, PA, USA; ${ }^{2}$ Biopsychology and Neuroscience Programs, Department of \\ Psychology, University of Michigan, Ann Arbor, MI, USA; ${ }^{3}$ Department of Neurobiology and Behavior, University of California, Irvine, CA, USA
}

\begin{abstract}
It is well known that the subthalamic nucleus (STN) plays an important role in regulating motor function, but recent studies suggest the STN is also involved in regulating motivated behavior. For example, bilateral lesions of the STN increase motivation for both food and cocaine as assessed by 'breakpoint' on a progressive ratio schedule. However, the psychological mechanism(s) by which STN lesions increase motivation for rewards is unknown. We hypothesized that STN lesions might influence one specific component of motivation, the attribution of incentive value (incentive salience) to reward-related cues. We tested this hypothesis by quantifying the ability of a discrete cue that had been paired with the non-contingent delivery of either food or cocaine to elicit approach towards it (ie, to produce a 'sign-tracking' conditioned response, CR). STN lesions made prior to training increased asymptotic levels of sign-tracking behavior directed towards a cue paired with either food or cocaine. In addition, when STN lesions were made after animals had already undergone Pavlovian training, and animals were tested under extinction conditions, the STN lesion still facilitated a sign-tracking CR. Finally, reintroduction of the US (food) following extinction immediately restored robust sign-tracking behavior in animals with STN lesions. We speculate, therefore, that the STN is part of a neural system that moderates the amount of incentive salience attributed to reward-related stimuli. Activity in this neural system may help mitigate the development of compulsive behavioral disorders, such as addiction, which are characterized by pathological control over behavior by reward-associated cues.

Neuropsychopharmacology (2008) 33, 2352-236I; doi:I0.1038/sj.npp. I301653; published online 5 December 2007
\end{abstract}

Keywords: autoshaping; incentive motivation; addiction; impulsivity; conditioned stimuli; classical conditioning

\section{INTRODUCTION}

Research on the subthalamic nucleus (STN) has focused on its role in movement disorders, such as Parkinson's and Huntington's disease (Albin et al, 1989; Crossman, 1990), but there is increasing evidence that the STN is also involved in regulating motivated behavior. For example, deep brain stimulation of the STN for the treatment of Parkinson's disease (Limousin et al, 1998; Charles et al, 2002; Welter et al, 2002), which is thought to inactivate the STN (Benazzouz et al, 1993, 1995, 2000; Lozano et al, 2002), produces non-motoric side-effects, including compulsive gambling (Lu et al, 2006; Smeding et al, 2007), increased desire for levadopa treatment (Houeto et al, 2002; Schupbach et al, 2005), and an increased disposition for addiction (Funkiewiez et al, 2003). Consistent with this, preclinical studies have shown that bilateral STN lesions increase motivation for both food (Baunez et al, 2002) and

*Correspondence: Dr JM Uslaner, Merck \& Co. Inc., 770 Sumneytown Pike, West Point, PA 19486, USA, Tel: + I 2156526617 ,

E-mail: Jason_Uslaner@merck.com

Received 5 September 2007; revised 18 October 2007; accepted 10 November 2007 cocaine (Uslaner et al, 2005), as measured by progressive ratio responding, and increase impulsive responding for a food reward, which could be indicative of enhanced motivation (Baunez et al, 1995; Baunez and Robbins, 1997; Phillips and Brown, 1999; Florio et al, 2001; Uslaner and Robinson, 2006).

Why STN lesions produce these effects is unknown, but we hypothesized that STN lesions may increase the incentive salience of cues associated with rewards (Uslaner et al, 2005; Uslaner and Robinson, 2006). Incentive salience attribution is a hypothetical process by which cues associated with rewards are posited to acquire incentive value, and thus the ability to elicit approach towards them, to energize ongoing instrumental actions, and to serve as reinforcers in their own right. The theoretical framework for this concept, which we use here, has been discussed extensively by Berridge and his colleagues (Robinson and Berridge, 1993; Berridge, 2001). One potential method for assessing the incentive value of a reward-related cue is to quantify its ability to elicit approach towards it (Berridge, 2001). When a discrete cue or 'sign' (conditioned stimulus; CS) is paired non-contingently with delivery of a natural reward such as food (unconditioned stimulus; US) or a drug of abuse such as cocaine, the cue can become attractive, 
eliciting approach towards it, a phenomenon known as sign-tracking (Brown and Jenkins, 1968; Jenkins and Moore, 1973; Tomie, 1996; Uslaner et al, 2006). Thus, the degree to which reward-related stimuli elicit approach, investigation and engagement (produce a sign-tracking CR) provides an index of the degree to which the cue has been attributed with incentive salience. Therefore, we used this procedure (sometimes called 'autoshaping', although this is a misnomer because no action is reinforced or 'shaped') to determine the effects of STN lesions on the ability of a stimulus paired with either a natural reward (food) or a drug reward (cocaine) to elicit approach and/or engagement. Furthermore, if STN lesions increase the attribution of incentive salience, rather than just facilitating Pavlovian learning, they should be effective in promoting approach to a rewardassociated cue whether made before or after Pavlovian training, and therefore we examined this variable as well.

\section{METHODS}

\section{Experiment 1: The Effects of STN Lesions on the Acquisition of Sign-Tracking Behavior to a Food-Paired CS}

Rationale. Experiment 1 was designed to examine whether STN lesions made prior to training influence the development of a sign-tracking CR to a food-paired CS.

Subjects. Thirty-two male Sprague-Dawley rats (Harlan) weighing 225-250 g were housed individually in clear square plastic cages and were given a 1-week acclimatization period before any experimental manipulation. The rooms were temperature and humidity controlled and on a $14 / 10 \mathrm{~h} \mathrm{light/dark}$ cycle (lights on at $0700 \mathrm{~h}$ ). Water was available ad libitum, whereas food was available ad libitum for the first 5 days of acclimatization, after which the animals were maintained on $18 \mathrm{~g}$ chow per day to keep their body weight at $90 \%$ of their free-feeding weight.

Surgery. Prior to training, animals were randomly chosen to receive sham $(n=11)$ or bilateral STN lesion surgery $(n=21)$. Surgical procedures were identical to those described in Uslaner et al (2005). Rats were anesthetized with ketamine hydrochloride $(75 \mathrm{mg} / \mathrm{kg}$ i.p.; Fort Dodge Animal Health, Fort Dodge, IA, USA) and xylazine hydrochloride $(7.5 \mathrm{mg} / \mathrm{kg}$ i.p.; Ben Venue Laboratories, Bedford, $\mathrm{OH}, \mathrm{USA}$ ) and secured into a stereotaxic apparatus. Twentyone rats received bilateral injections of ibotenic acid $(12.5 \mu \mathrm{g} / \mu \mathrm{l}$ in $0.1 \mathrm{~m}$ phosphate-buffered saline ( $\mathrm{pH} 7.4)$; Tocris Cookson Inc., Ellisville, MO, USA) and the remaining 11 rats received vehicle alone $(0.1 \mathrm{M}$ phosphate-buffered saline). The coordinates targeting the STN were measured from bregma: anterior/posterior, $3.8 \mathrm{~mm}$; lateral, $\pm 2.4 \mathrm{~mm}$; dorsal/ventral, $8.35 \mathrm{~mm}$ (from skull). The volume injected was $0.5 \mu \mathrm{l}$ per side infused over 3 min using a $10-\mu \mathrm{l}$ Hamilton syringe connected by tubing fitted to a 30 -gauge $(0.254 \mathrm{~mm}$ in diameter) stainless steel injector needle. A micropump was calibrated to deliver the exact volume of fluid.

Testing Apparatus. Behavioral testing was conducted in standard operant chambers (Medical Associates Inc.,
Georgia, VT, USA) containing an acrylic hinged loading door, stainless steel side panels and an acrylic back panel $(22 \times 18 \times 13 \mathrm{~cm})$. The chambers were located in soundand light-attenuating cabinets equipped with fans providing constant ventilation and low-level background noise, and a house light providing illumination. Centered on one wall of the chamber was a food cup into which $45 \mathrm{mg}$ bananaflavored pellets could be delivered. In addition, two levers that could be extended and retracted were positioned to the left and right of the food cup. When extended, the levers were approximately $3 \mathrm{~cm}$ above the floor. There was also a stimulus light located behind each lever (lever light), which illuminated the lever only when it was extended.

Training. On the first day of training, STN-lesioned animals and sham controls were placed in the operant chambers and given banana pellets on a random interval schedule with a mean inter-trial interval of $30 \mathrm{~s}$ to familiarize the animals with retrieving the pellets from the food cup. This session lasted $\sim 25 \mathrm{~min}$ (after 50 pellets were delivered).

On the second day of training, animals continued to receive banana pellets on a random interval schedule with a mean inter-trial interval of $30 \mathrm{~s}$, but prior to each pellet presentation one lever ( $\mathrm{CS}+$; right or left, counterbalanced between groups) was extended for $8 \mathrm{~s}$. The second lever, serving as a control ( $\mathrm{CS}-$ ) was extended on a random interval $30 \mathrm{~s}$ schedule, but was explicitly unpaired with pellet presentation. It is important to note that receipt of banana pellets was not contingent on lever pressing, but that the pellet was presented following the CS + regardless of how the animal behaved. Under these conditions animals often come to approach and contact the CS + lever (typically biting it), and therefore, the latency to initially depress the lever and total lever depressions were recorded to assess the tendency to approach and engage the CS + (Jenkins and Moore, 1973). Animals were given $25 \mathrm{CS}+$ trials and $25 \mathrm{CS}-$ trials ( $\mathrm{CS}+$ and $\mathrm{CS}-$ trials randomized) per daily test session, for a total of 21 sessions (days).

\section{Experiment 2: The Effects of STN Lesions on the Acquisition of Sign-Tracking Behavior to a Cocaine- Paired CS}

Rationale. The results from experiment 1 indicated that STN lesions increased sign-tracking to a CS paired with food. Experiment 2 was designed to examine whether STN lesions made prior to training also increase sign-tracking behavior to a CS paired with an intravenous injection of cocaine.

Subjects. Forty-two male Sprague-Dawley rats (Harlan) weighing 225-250 g were housed and handled in the same way as in experiment 1 except that the animals were not food-restricted.

Surgery. Prior to training, animals were randomly chosen to receive sham $(n=17)$ or bilateral STN lesion surgery $(n=$ 25). Surgical procedures were identical to those in experiment 1.

Ten to twelve days following STN surgery animals underwent catheter surgery, in which an intravenous catheter was implanted into the animal's right jugular vein. Catheter 
construction and implantation were based on procedures described previously (Weeks, 1972; Crombag et al, 2000). Briefly, one end of a silicone catheter was inserted into the right external jugular vein and the other was passed subcutaneously to exit the back of the animal, where it was connected to a pedestal constructed from 22 gauge cannula connected to a piece of polyethylene mesh using dental cement. Following surgery, catheters were flushed daily with $0.1 \mathrm{ml}$ sterile saline containing gentamicin $(0.08 \mathrm{mg} / \mathrm{ml})$ to prevent occlusions and microbial buildup in the catheter. Both before and after training catheters were screened for patency by manually injecting $0.1 \mathrm{ml}$ of the short-acting barbiturate sodium thiopenthal (i.v.; $20 \mathrm{mg} / \mathrm{ml}$ in sterile water). Rats that became ataxic within $5 \mathrm{~s}$ were considered to have patent catheters. Only rats with patent catheters were included in the analysis.

Testing apparatus. Behavioral testing was conducted in identical operant chambers as described above, however the chambers were outfitted differently. Two illuminated levers that could be extended and retracted were positioned to the far left and right of the front wall. For some animals lever presentation (left-right counterbalanced) was explicitly paired with an injection of cocaine, whereas for other animals lever presentation was explicitly unpaired with cocaine (see below for details). Thus, individual animals received either presentation of a $\mathrm{CS}+$ or a $\mathrm{CS}-$, but no animals received both. The reason for not including both CS + and CS- presentations using a within-subjects design in this experiment, and thus examining discriminated approach, was because of the duration of action of cocaine. After an injection it is important for the effect of the US (cocaine) to have dissipated before presentation of the next CS-US pairing (Uslaner et al, 2006), and a within-subjects design involving both $\mathrm{CS}+$ and $\mathrm{CS}-$ presentations resulted in very long test sessions. Therefore, independent groups were used to examine the effect of a $\mathrm{CS}+v s \mathrm{CS}-$ on approach behavior. The chamber was further modified in this experiment to include a plastic transluscent insert that formed two short corridors $(16.5 \mathrm{~cm}$ high $\times 5.5 \mathrm{~cm}$ wide $\times$ $5.5 \mathrm{~cm}$ deep) allowing access to the levers. The plastic insert provided support for photocells that were aligned $1 \mathrm{~cm}$ in front of the extended lever, allowing for the automated recording of approaches to the lever. We previously found that when paired with an intravenous injection of cocaine, animals approach and investigate the lever, but seldom touch it, and therefore lever presses do not provide a good measure of approach behavior when cocaine is used as the US (Uslaner et al, 2006). A similar phenomenon has been described when rewarding intracranial brain stimulation is used as the US (Peterson et al, 1972). Thus, in this experiment an approach was recorded when an animal broke the photocell beam located just in front of the extended lever.

Habituation. We previously found that rats tend to approach the illuminated lever at a high rate during the first few training sessions, independent of drug administration, presumably because of its novelty (Uslaner et al, 2006). Thus, to decrease baseline, responding animals were first habituated to the presentation of the illuminated lever and sound of the infusion pump. Habituation sessions were initiated by activating the house light and white noise generator, both of which remained on throughout the session. Habituation sessions consisted of 30 individual trials in which the illuminated lever was extended for $8 \mathrm{~s}$ and the infusion pump activated for $2.8 \mathrm{~s}$. The inter-trial interval varied randomly with a mean interval of $120 \mathrm{~s}$. An entire session lasted approximately $1 \mathrm{~h}$.

Testing. Following habituation animals that had received STN lesions or sham lesions were randomly divided into four groups. Animals from all groups were brought to the test chambers and connected to infusion lines. Sessions began with the activation of the red house light and white noise generator. Animals were then given eight trials, with a randomly varying inter-trial interval (mean of $900 \mathrm{~s}$; each session lasted $\sim 120 \mathrm{~min})$. For some of the animals that had received STN lesions or sham surgery (paired lesion and paired sham; $n=18$ and 12 respectively), each lever presentation (lasting $8 \mathrm{~s}$ ) was paired with a non-contingent intravenous injection of $0.2 \mathrm{mg} / \mathrm{kg}$ of cocaine (weight of the salt, dissolved in $0.9 \%$ saline). The infusion pump was activated upon insertion of the lever, because of the delay involved with an injection, and the injection itself took $2.8 \mathrm{~s}$. The other two groups consisted of lesioned and sham animals (unpaired lesion and sham; $n=7$ and 5 respectively) that received non-contingent infusions of $0.2 \mathrm{mg} / \mathrm{kg}$ cocaine that were explicitly not paired with the presentation of the lever (in this group cocaine was administered $2 \mathrm{~min}$ after retraction of lever). The dose of cocaine was chosen because we had previously found that $0.3 \mathrm{mg} / \mathrm{kg}$ produced robust sign-tracking behavior in normal animals and we hypothesized that animals with STN lesions would be even more sensitive to the effects of cocaine. Thus, we chose to use a lower dose of cocaine to avoid a ceiling effect that could mitigate the detection of between group differences. The number of approaches out of the eight lever presentations per session as well as the latency to approach the lever was recorded. Blocks of two consecutive sessions' data were combined for analysis because of the variability inherent in only presenting the lever eight times per session. Animals were tested for 20 sessions.

\section{Experiment 3: The Effects of STN Lesions Given After the Acquisition of Sign-Tracking to a Food-Paired Stimulus: Responding during Extinction and Re-acquisition}

Rationale. The results from experiments 1 and 2 indicated that STN lesions increased sign-tracking to a CS paired with either a natural reinforcer (food) or a drug of abuse (cocaine). However, from these two experiments alone it is difficult to determine whether STN lesions produced their effects by facilitating associative learning or by enhancing the incentive value of the CS. If STN lesions increase incentive salience attribution, rather than facilitating learning, then they should increase a sign-tracking CR even when made after the sign-tracking CR is acquired. Experiment 3 was designed to dissociate these two competing possibilities. Animals were first trained to sign-track a CS paired with food and only after the acquisition of a stable signtracking CR were they given STN lesions. We then examined the impact of the STN lesion on the sign-tracking CR 
when animals were subsequently tested under extinction conditions, and thus no further CS-US pairings occurred. If STN lesions only facilitated Pavlovian learning then a lesion made after the development of asymptotic performance should have no impact on subsequent performance during extinction (or might increase the rate of extinction, given that extinction is a form of learning). On the other hand, if STN lesions increased the incentive value of the $\mathrm{CS}+$, then the lesions should immediately impact performance during extinction. Furthermore, experiment 3 also examined the influence of STN lesions on the re-acquisition of signtracking once extinction had occurred, as well as the influence of lesions made $2 \mathrm{~cm}$ dorsal to the STN, to ensure that the effects of STN lesions were due to damage confined to the STN.

Subjects. Forty-four male Sprague-Dawley rats (Harlan) weighing 225-250 g were housed and handled in the same way as in experiment 1 .

Testing apparatus. Behavioral testing was conducted in identical chambers as those used in experiment 1.

Training prior to surgery. Animals were trained to signtrack in an identical manner as in experiment 1. Importantly, this training occurred prior to surgery, unlike in experiment 1 in which the training occurred after surgery.

Surgery. After training, animals were randomly chosen to receive sham $(n=12)$, bilateral STN lesions $(n=19)$, or lesions $2 \mathrm{~cm}$ dorsal to the STN $(n=11)$. This later group was included to ensure that the observed effects were due to damage of the STN, as opposed to surrounding structures. Surgical procedures were identical to those described in experiment 1 .

Extinction sessions following surgery. Ten to twelve days following surgery, animals were tested under identical conditions as during training, other than that food pellets were not dispensed during the session. Extinction sessions were carried out for 7 days.

Reacquisition of sign-tracking. Following 7 days of extinction training, animals were reexposed to the initial Pavlovian training conditions for an additional 10 sessions (ie, food was dispensed following each CS + presentation).

\section{Experiment 4: The Effects of STN Lesions Given After Acquisition of Sign-Tracking to a Food-Paired Stimulus: Influence of Exposure to the US Prior to Extinction Training}

Rationale. The results from experiment 3 indicated that STN lesions had a significant but modest effect on responding during extinction, but a very large effect on responding when the US was again introduced during retraining. Experiment 4 was designed to examine whether STN lesions might also directly impact the incentive value of the food reward itself (the US). Thus, as in experiment 3 , animals in experiment 4 were trained to sign-track prior to receiving STN lesions, and following surgery were tested under extinction conditions. However, prior to extinction training animals were given banana pellets in their home cage so that they could experience the US in their new state (ie, without an STN). We hypothesized that if STN lesions enhanced the incentive value of the US, then exposure to the now magnified US should lead to a transfer of increased value to the $C S$, resulting in a greater sign-tracking $C R$ during extinction.

Subjects. Thirty-eight male Sprague-Dawley rats (Harlan) weighing 225-250 g were housed and handled in the same way as in experiment 1 .

Testing apparatus. Behavioral testing was conducted in identical chambers as those used in experiment 1.

Training prior to surgery. Animals were trained in an identical manner as in experiments 1 and 3 . As in experiment 3, this training occurred prior to surgery.

Surgery. After training, animals were randomly chosen to receive sham surgery $(n=17)$ or bilateral STN lesions $(n=21)$. Surgical procedures were identical to those described in experiments 1 and 3.

Exposure to the US following surgery. Two and one day prior to extinction sessions animals were given 25 banana flavored pellets in their home cages.

Testing following surgery. Ten to twelve days following surgery, animals were tested under extinction conditions identical to that described for experiment 3 .

Statistical analysis. Data were analyzed using 2-way analyses of variance (ANOVA) with a repeated measures factor (day) and between subjects factor (group). For studies in which banana pellets were the US the two dependent variables analyzed were difference scores (depressions of the CS + lever minus the CS- lever), reflecting discriminated CS + approach, and latency to contact the CS + Animals that were considered goal-trackers (those that made greater number of approaches to the food cup than the CS + lever during CS + presentation on the final day of testing) were excluded from all analyses (Flagel et al, 2007). STN lesions did not appear to influence whether an animal became a goal- or sign-tracker (in experiment 1 there were 6 goal-trackers, 3 received sham surgery and 3 had bilateral STN lesions). In experiment 2 there was no obvious location associated with the reward, so the identification of goaltrackers was not possible. In experiments 3 and 4, surgery was conducted after training, so did not influence the development of goal- $v s$ sign-tracking. For studies in which drug was the US, the two dependent variables analyzed were $\mathrm{CS}+$ approaches ( $\max 16)$ and latency to approach the CS + . In addition, in experiments 1 and 2, we were interested in not only whether there were group differences in the acquisition of sign-tracking, but also in asymptotic performance. To assess the latter we averaged responding during the final three test sessions and tested for group differences using a planned $t$-test (Exp. 1) or a one-way ANOVA followed by post hoc comparisons (Fischer's PLSD; Exp. 2). 


\section{RESULTS}

\section{Experiment 1: STN Lesions Increase Sign-Tracking Behavior Directed Toward a Food-Paired CS +}

Histology. Figure 1 shows a representative section from an animal with an STN lesion and a sham control. STNlesioned animals showed a loss of neurons that was mainly restricted to the STN, with little damage to structures outside the STN. Nine animals were excluded because there was either no detectable cell loss or the damage extended outside the STN.

Sign-tracking. With training both sham controls and animals with STN lesions developed a sign-tracking CR that had a topography exactly as described in the literature (Davey and Cleland, 1982). Upon cue presentation, the animals would initially orient to the CS (lever) and then rapidly approach and engage it. Contact with the lever did not consist of 'pressing' it with the paws, but of grasping, gnawing and biting it, and the more vigorously the animal engaged the lever in this way the greater the number of lever 'presses' recorded. As has been pointed out many times, the nature of this sign-tracking $\mathrm{CR}$ resembles an attempt to 'ingest' the CS, and it has been suggested that this is because the CS takes on some of the properties of the US (food in this case). When the US consists of a reward other than food the topography of the CR is appropriate to that US; eg, if water is used as the reward rats may lick the lever (Jenkins and Moore, 1973; Davey and Cleland, 1982; Davey et al, 1984).

Thus, with training both groups increased the number of contacts with the $\mathrm{CS}+$, relative to the CS- (difference scores; main effect of session; $p<0.001$ ), however, there was also a significant session by group interaction (Figure $2 ; \mathrm{F}_{(20,300)}=$ $1.62, p=0.047)$. This was because animals with STN lesions showed greater asymptotic levels of sign-tracking behavior. Indeed, difference scores during the last three days of training were significantly higher in STN-lesioned animals, relative to sham controls (Figure 2; $t_{(15)}=2.61, p=0.02$ ).

The latency data further confirmed that the CS + had greater incentive value in STN-lesioned animals, relative to sham controls. Thus, both groups approached the CS+ more quickly as a function of session $\left(\mathrm{F}_{(20,300)}=6.83\right.$, $p=0.02)$, and there was a trend for the STN-lesioned animals to approach more quickly than the sham controls (Figure 2; main effect of group; $\mathrm{F}_{(1,15)}=3.12, p=0.097$; group $\mathrm{X}$ session, $\left.\mathrm{F}_{(20,300)}=0.57, p=0.93\right)$. Indeed, during the last 3 days of testing, when performance had reached asymptote, the STN lesion group approached the CS+ significantly more quickly than did the control group (Figure $2 ; t_{(15)}=2.50, p=0.027$ ).

\section{Experiment 2: STN Lesions Increase Sign-Tracking Behavior Directed Towards a Cocaine-Paired CS}

Histology. Examination of the tissue from experiment 2 revealed very similar cell loss as that observed in experiment 1 . Ten animals had to be excluded from experiment 2 either because there was insufficient damage to the STN, cell loss occurred outside of the STN, or catheter patency was lost either before testing or was discovered after testing was completed.

Sign-tracking. STN-lesioned animals and sham controls who received the CS unpaired with cocaine did not differ in

Sign-tracking to a Food-Paired CS+ When the Lesion is Made Prior to Training
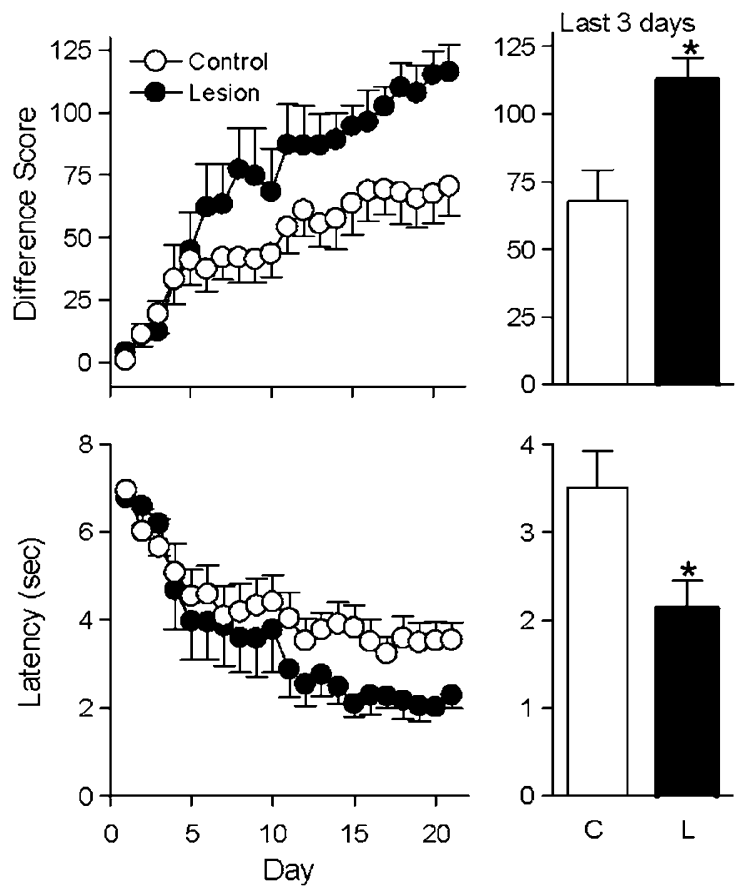

Figure 2 Influence of bilateral STN lesions on sign-tracking for a foodpaired CS + . STN lesions increased the level of sign-tracking for the foodpaired CS + as indicated by a significant increase in CS + contacts, relative to CS- (difference scores; top two panels), and as indicated by a shorter latency to contact the CS + (bottom two panels). *A significant difference relative to control.
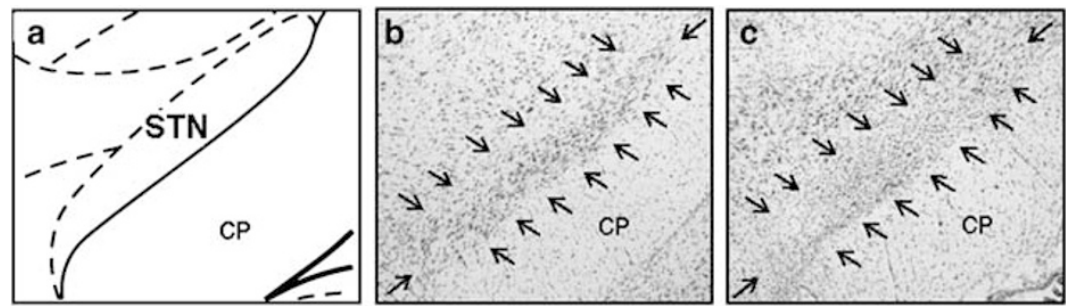

Figure I (a) Illustration showing the subthalamic nucleus (STN) bordering the cerebral peduncle (CP). (b) Representative cresyl-violet stained section at the level of the STN in a sham control. Arrows indicate the border of the STN and the CP is labeled to aid anatomical location. (c) Cresyl violet-stained section of the STN from a lesioned animal. Gliosis and cell loss are apparent in the section from the lesioned animal. 
the number of approaches made or latency to approach the $\mathrm{CS}$, and thus were combined to form one 'Unpaired' group (data not shown).

The topography of the sign-tracking CR when cocaine was used as the US was different than that for food, as we have described previously (Uslaner et al, 2006). Animals oriented to, approached and investigated (sniffed) the lever CS, but did not reliably touch it. The topography of this CR is exactly what has been described when electrical stimulation of the brain is used as the rewarding US (Peterson et al, 1972), and it is why lever presses could not be used as in index of approach behavior in this experiment (see Methods).

When the number of approaches to the CS + for all three groups were analyzed in an overall ANOVA, there was a main effect of group, which was because animals with bilateral STN lesions made significantly more CS + approaches relative to both the unpaired and sham-paired groups (Figure 3; main effect of group, $\mathrm{F}(1,30)=8.89$, $p=0.006$ and $\mathrm{F}_{(1,29)}=4.32, p=0.047$ respectively; group $\mathrm{X}$ block interactions NS). In contrast, the sham-paired group and the unpaired group did not differ from one another. Furthermore, there was a main effect of group on asymptotic level of performance (last 3 blocks; $\mathrm{F}_{(2,35)}=4.97$, $p=0.013$ ), which was because animals with STN lesions approached the CS + significantly more than either the unpaired group or the sham-paired group (Figure 3; Fischer's PLSD, $p=0.004$ and 0.046 , respectively).

STN lesions also increased how rapidly animals approached the $\mathrm{CS}+$, as indicated by a main effect of group and a group X block interaction. Animals with bilateral STN lesions approached the $\mathrm{CS}+$ more quickly than both the

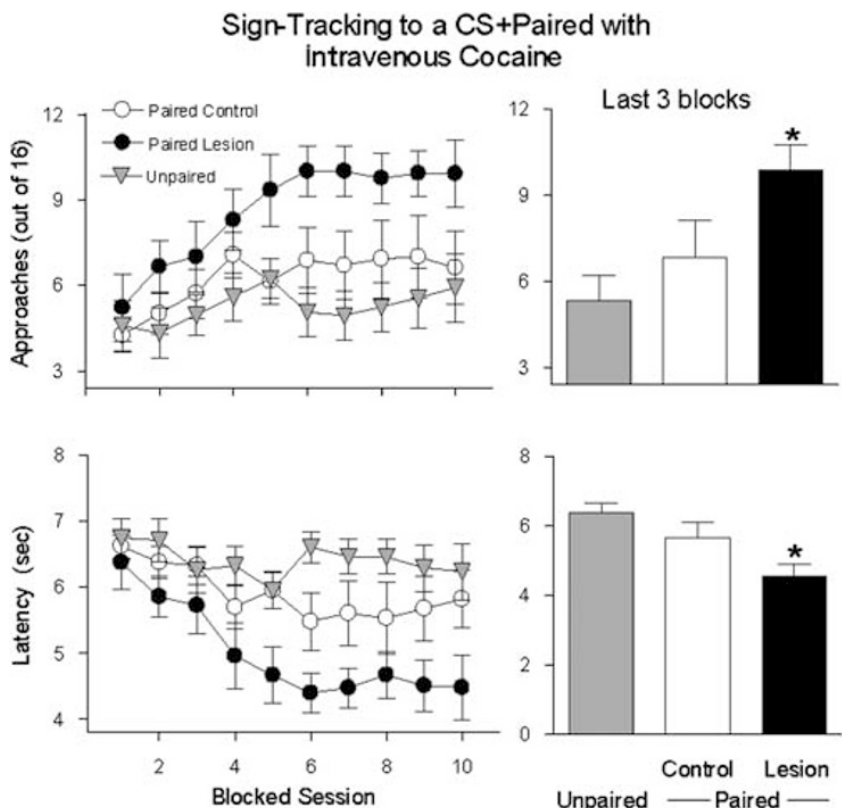

Figure 3 Influence of bilateral STN lesions on sign-tracking for a CS + paired with intravenous cocaine. STN lesions increased the level of signtracking for the drug-paired CS +, relative to both the unpaired group and the sham controls, as indicated by a significant increase in CS + approaches (top panels) and a decrease in the latency to approach the CS + (bottom panels). *A significant difference relative to both unpaired and control paired groups. unpaired group (Figure 3; main effect of group, $\mathrm{F}_{(1,30)}=8.89$, $p=0.003$; group X block interaction $\mathrm{F}_{(9,239)}=3.34, p=0.001$ ) and tended to approach the CS + more quickly than the sham-paired group (Figure 3; main effect of group, $\mathrm{F}_{(1,29)}=$ 4.04, $p=0.054$; group $\mathrm{X}$ block interaction, $\mathrm{F}_{(9,229)}=$ $0.58, p=0.81)$. The latency for the sham-paired group and the unpaired group to approach the CS + did not differ $\left(\mathrm{F}_{(1,34)}=2.33, p=0.136\right)$. In addition, there were significant group differences in the latency to approach the lever after performance had reached asymptote (last 3 blocks; $F_{(2,35)}=$ $5.98, p=0.006$ ), which was because animals with STN lesions approached the CS + significantly more quickly than either the unpaired group or the sham-paired group (Figure 3; Fischer's PLSD, $p=0.002$ and 0.043 , respectively).

\section{Experiment 3: STN Lesions Made After Training Increase Sign-Tracking during Extinction and Facilitate The Re-Acquisition of a Sign-Tracking CR}

Histology. Examination of the tissue from experiment 3 revealed very similar cell loss as that observed in earlier experiments. Nine animals had to be excluded from experiment 3 either because there was insufficient damage to the STN or cell loss occurred outside of the STN.

Training. Animals that were to later receive STN lesions and control subjects (dorsal controls and shams) did not differ in their preoperative performance (data not shown).

Sign-tracking during extinction. Animals that received sham surgery and dorsal lesion controls did not differ on any measure and thus were combined into a single control group (data not shown).

STN lesions made after training tended to increase difference scores during extinction, as revealed by a trend towards an interaction between group and session $\left(F_{(6,90)}=2.08, p=0.063\right)$. This was because STN-lesioned animals had greater difference scores during the first couple of extinction sessions, but not during the later sessions (Figure 4). A similar picture emerged for latency. Thus, there was a significant group $\mathrm{X}$ session interaction $\left(\mathrm{F}_{(6,90)}=2.83, p=0.014\right)$, which was because STN lesions decreased the latency to contact the $\mathrm{CS}+$ during the first couple of extinction sessions, relative to control animals (Figure 4).

Re-acquisition of sign-tracking. When food was reintroduced STN-lesioned animals immediately re-acquired robust sign-tracking behavior, and had a significantly greater CS difference score than controls (main effect of group, $\mathrm{F}_{(1,15)}=10.11, p=0.006$; group $\mathrm{X}$ session interaction, $\left.\mathrm{F}_{(10,150)}=0.66, p=0.76\right)$ and shorter latency to approach the $\mathrm{CS}+$ (main effect of group, $\mathrm{F}_{(1,15)}=7.83$, $p=0.013$; group $\mathrm{X}$ session interaction, $\mathrm{F}_{(10,150)}=0.5$, $p=0.89$; Figure 4 ).

\section{Experiment 4: STN Lesions Made After Training Increase Sign-Tracking During Extinction: Effect of Exposure to the Food US Prior to Testing}

Histology. Examination of the tissue from experiment 4 revealed very similar cell loss as that observed in earlier 

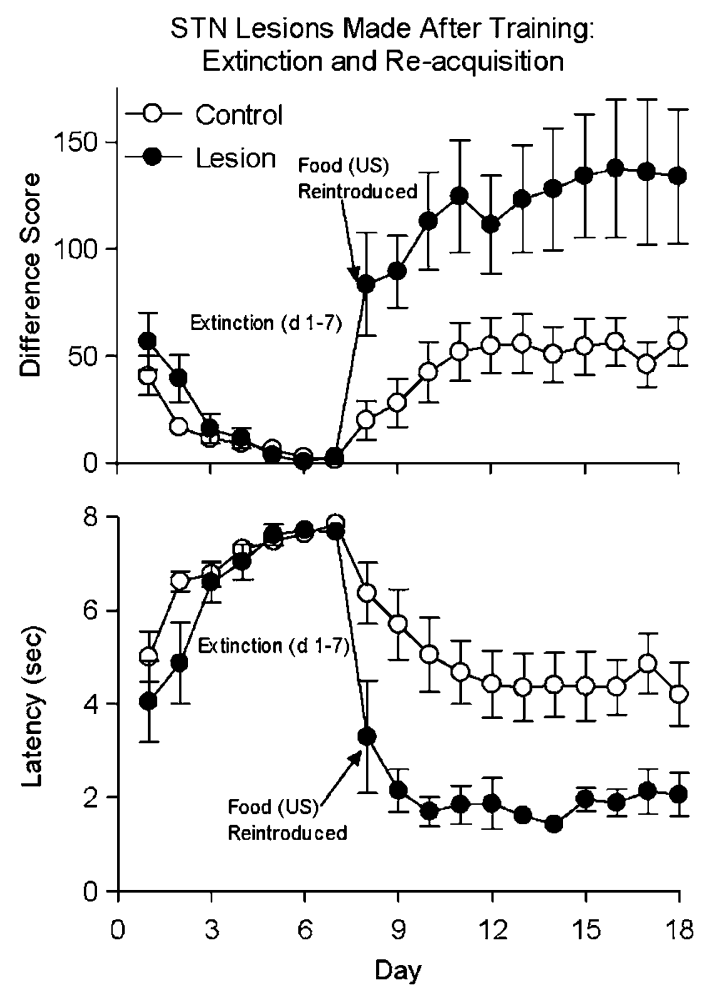

Figure 4 Influence of bilateral STN lesions on sign-tracking during extinction and re-acquisition for a food-paired CS + when training was conducted prior to surgery. During extinction, STN lesions had a small but significant effect on difference scores during the first several sessions of extinction (top panel), and tended to decrease the latency to approach the CS + during these first several sessions (bottom panel). When food was reintroduced, STN-lesioned animals quickly reacquired sign-tracking at levels significantly higher than sham controls, as reflected by greater CS difference scores and decreased latencies.

experiments. Six animals had to be excluded either because there was insufficient damage to the STN or cell loss occurred outside of the STN.

Training. Animals that were to later receive either STN lesion or sham surgery did not differ in their preoperative performance (data not shown).

Sign-tracking during extinction following exposure to the US prior to extinction. An examination of difference scores during testing under extinction conditions revealed that STN lesions significantly increased sign-tracking behavior, as indicated by an increase in CS difference scores (main effect of group, $\mathrm{F}_{(1,16)}=5.43, p=0.033$; group $\mathrm{X}$ day interaction, $\left.\mathrm{F}_{(6,96)}=1.76, p=0.116\right)$ and a decrease in latency to contact the CS + (main effect of group, $\mathrm{F}_{(1,16)}=5.22$, $p=0.036$; group $\mathrm{X}$ day interaction, $\mathrm{F}_{(6,96)}=0.47, p=0.83$; Figure 5). Thus, when animals were exposed to the US prior to testing under extinction conditions animals with STN lesions continued to approach the CS + more than did control animals.

\section{DISCUSSION}

When a discrete cue is paired with the delivery of a reward the cue is believed to acquire incentive value (incentive

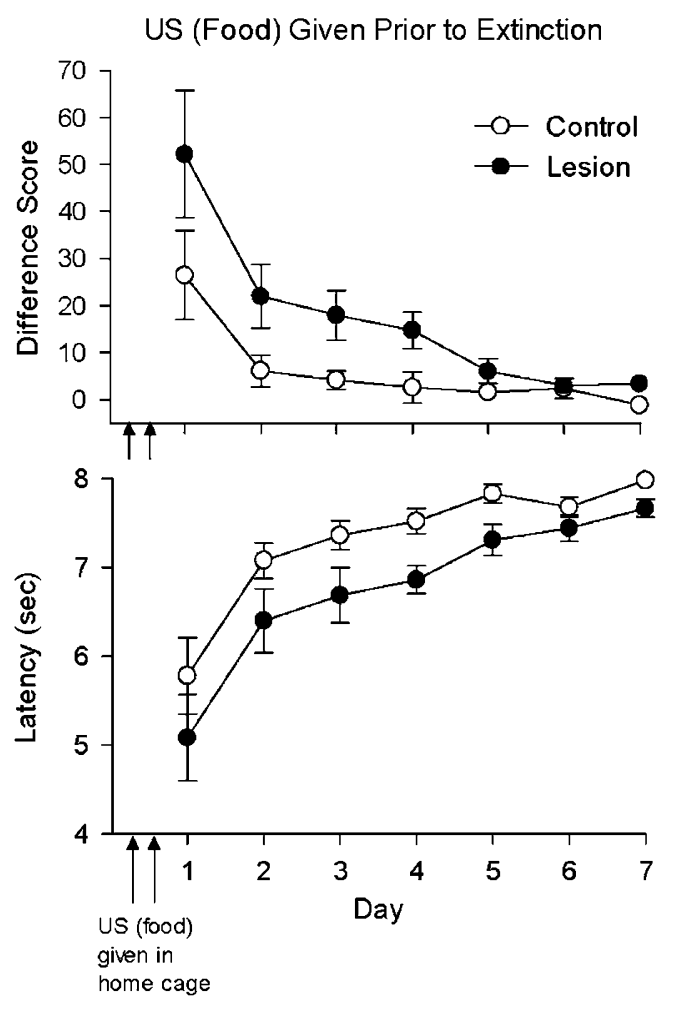

Figure 5 Influence of bilateral STN lesions on sign-tracking during extinction after animals had been exposed to the US prior to extinction sessions. STN lesions significantly increased sign-tracking during extinction, as revealed by a significant effect of lesion on both CS difference scores and latency to contact the CS +.

salience) via Pavlovian learning, and one property of cues that have been attributed with incentive salience is that they elicit approach towards them; that is, they can act as 'motivational magnets' (Berridge, 2001). This property is evident in the phenomenon of sign-tracking, whereby reward-related cues ('signs') can come to be so irresistibly attractive that they continue to elicit approach even when it results in diminished reward (Williams and Williams, 1969; Hearst and Jenkins, 1974). We hypothesized that the STN is part of a neural system that moderates the attribution of incentive salience to reward-related cues (Uslaner et al, 2005; Uslaner and Robinson, 2006), and to explore this hypothesis we studied the effects of STN lesions on a signtracking conditioned response (CR) directed towards cues associated with delivery of either a natural reward (food) or a drug of abuse (cocaine). We report that when made either before or after Pavlovian training in which presentation of a lever CS was paired with a reward (US), STN lesions increased the tendency of rats to approach the CS; that is, to show a sign-tracking CR.

There are at least two different interpretations as to why STN lesions increased sign-tracking behavior in the experiments in which the lesions were made prior to training. One is that the lesion directly facilitated Pavlovian learning. There are, however, a number of reasons to think that STN lesions did not directly facilitate learning of the CS-US association. First, examination of Figures 2 and 3 shows that STN lesions made prior to training did not influence responding during the first 4-5 days of training, as the CR 
developed. In fact, the development of the sign-tracking CR initially developed at the same rate in both groups. Instead, group differences were primarily due to differences in asymptotic levels of performance. Second, and even more importantly, when STN lesions were made after the animals had already been trained, the lesions facilitated a signtracking CR even when animals were tested under extinction conditions, when no additional pairings between the CS and US occurred. That is, in experiments 3 and 4 there was no opportunity for the lesion to strengthen the CS-US association because the CS was presented during extinction, in the absence of the US (for a similar argument, see Wyvell and Berridge, 2001; Tindell et al, 2005). This is similar to what we have seen in previous studies, in which STN lesions immediately increased impulsive responding on a DRL-30s schedule and immediately increased the value of large rewards, relative to small rewards (Uslaner and Robinson, 2006). Third, in experiment 3 reintroduction of the US during retraining immediately induced much more robust sign-tracking in animals with STN lesions than in controls, an effect that is not easily interpretable as increased learning.

Rather than a direct impact on learning, we suggest that the effects of STN lesions may be interpreted within a theoretical framework posited by Berridge and his colleagues (Robinson and Berridge, 1993; Berridge, 2001). By this interpretation, STN lesions may have increased the amount of incentive salience attributed to the CS and/or the US (or more accurately, to the representation of the CS or US). That is, we suggest that STN lesions may have altered a motivational rather than a learning process (acknowledging, of course, that alterations in the former can influence the latter). It is difficult to dissociate learning from motivational explanations in experiments in which a lesion is made prior to training, as in experiments 1 and 2 . Nevertheless, even in these experiments a motivational interpretation is consistent with the fact that the primary effect of STN lesions was on asymptotic levels of performance rather than on the initial development of the CR. Furthermore, the impact of STN lesions made after training, when testing was conducted during extinction, and when there were no further CS-US pairings, is readily explicable by the hypothesis that the lesion increased the incentive value of the CS (and/or the US).

The hypothesis that STN lesions increase incentive salience attribution can also explain the effects of STN lesions in other situations. Lesions or pharmacologicallyinduced inactivation of the STN has been reported to produce each of the following psychological phenomena: increased 'impulsive action' (Baunez et al, 1995; Baunez and Robbins, 1997; Phillips and Brown, 1999; Florio et al, 2001; Uslaner and Robinson, 2006), decreased 'impulsive choice' (Winstanley et al, 2005; Uslaner and Robinson, 2006), increased motivation (Baunez et al, 2002; Uslaner et al, 2005), and increased perseveration (Baunez and Robbins, 1997, 1999). Although each of these psychological constructs might truly be independent, the behavioral procedures used to study these processes are influenced by how much incentive value is attributed to a CS, including the response manipulandum and other stimuli associated with rewards (Tomie, 1996). For example, if the incentive value of the manipulandum were increased as a result of an STN lesion an animal may have more difficulty withholding a response (ie, the incentive properties of CS would increase the likelihood that the animal would respond), and animals might make what appear to be 'impulsive actions'. The same logic can be applied to the findings that STN lesions increase motivation as assessed by 'breakpoint' on a progressive ratio schedule (Baunez et al, 2002; Uslaner et al, 2005), facilitate delay-discounting (Winstanley et al, 2005; Uslaner and Robinson, 2006), and increase perseverative responding (Baunez and Robbins, 1997, 1999). Finally, this interpretation is also consistent with reports that deep brain stimulation of the STN for the treatment of Parkinson's disease, which may inactivate the structure, sometimes produces compulsive behavioral disorders (see Introduction for references).

Indeed, this view of the effect of STN lesions might also clarify the results of one report that seems discrepant with our findings. Winstanley et al (2005) reported that STN lesions disrupted the acquisition of sign-tracking to a food-paired CS, the opposite of what we found. However, in the Winstanley et al (2005) study animals were trained and tested using a delay-discounting procedure prior to Pavlovian training using a sign-tracking procedure, and the same US was used in the delay-discounting and signtracking studies. Thus, these animals had presumably formed a variety of CS-US associations prior to Pavlovian training. If STN lesions increase the incentive value of CSs, thereby indirectly facilitating learning, it might be more difficult for STN-lesioned animals to acquire new associations when switched to a different task in similar surroundings. Of course other procedural factors, such as the size of the lesions or strain of rat used, might account for the discrepant results as well (see Uslaner et al, 2005).

In conclusion, we report that STN lesions facilitate a sign-tracking CR evoked by discrete cues associated with the non-contingent presentation of either food or cocaine, and hypothesize that this is because STN lesions increase the incentive salience attributed to reward-associated cues. We speculate, therefore, that one function of the intact STN is to moderate how much incentive salience is attributed to reward-related cues. Of course, the attribution of incentive salience to stimuli associated with rewards is usually adaptive because such signs generally signal the location and availability of rewards important for survival, such as food, and it is usually desirable to approach such cues. However, if cues become imbued with excessive incentive salience this may lead to maladaptive behavior. Indeed, under some circumstances cues can become so irresistible that they continue to control behavior even when it is disadvantageous, in that continued approach or engagement leads to loss of the reward (Breland and Breland, 1961; Williams and Williams, 1969; Hearst and Jenkins, 1974). In this way the attribution of pathological levels of incentive salience to cues may contribute to the development of compulsive behavioral disorders, including addictions (Newlin, 1992; Robinson and Berridge, 1993; Tomie, 1996; Uslaner et al, 2006; Flagel et al, 2008). In addition, it may also be necessary to dampen reactivity to rewardrelated cues for higher cognitive systems to gain control over actions and decisions. It is surely not always adaptive to act automatically in response to powerful incentive stimuli (as often occurs in addiction), and thus it is 
probably important that the brain not only have a system to attribute incentive salience to reward-associated cues but also a system that moderates that process. We suggest that the STN may comprise part of the latter system and as such may serve as an important target for therapies aimed at treating compulsive behavioral disorders.

\section{ACKNOWLEDGEMENTS}

This research was supported by Grant R37 DA04294 from the National Institute on Drug Abuse to TER. We thank Drs Kent Berridge and Shelly Flagel for helpful comments on an earlier draft of the article.

\section{DISCLOSURE/CONFLICT OF INTEREST}

We declare that, except for income received from our primary employer, no financial support or compensation has been received from any individual or corporate entity over the past 3 years for research or professional service and there are no personal financial holdings that could be perceived as constituting a potential conflict of interest.

\section{REFERENCES}

Albin RL, Young AB, Penney JB (1989). The functional anatomy of basal ganglia disorders. Trends Neurosci 12: 366-375.

Baunez C, Amalric M, Robbins TW (2002). Enhanced food-related motivation after bilateral lesions of the subthalamic nucleus. J Neurosci 22: 562-568.

Baunez C, Nieoullon A, Amalric M (1995). In a rat model of Parkinsonism, lesions of the subthalamic nucleus reverse increases of reaction time but induce a dramatic premature responding deficit. J Neurosci 15: 6531-6541.

Baunez C, Robbins TW (1997). Bilateral lesions of the subthalamic nucleus induce multiple deficits in an attentional task in rats. Eur J Neurosci 9: 2086-2099.

Baunez C, Robbins TW (1999). Effects of transient inactivation of the subthalamic nucleus by local muscimol and APV infusions on performance on the five-choice serial reaction time task in rats. Psychopharmacology (Berl) 141: 57-65.

Benazzouz A, Gao DM, Ni ZG, Piallat B, Bouali-Benazzouz R, Benabid AL (2000). Effect of high-frequency stimulation of the subthalamic nucleus on the neuronal activities of the substantia nigra pars reticulata and ventrolateral nucleus of the thalamus in the rat. Neuroscience 99: 289-295.

Benazzouz A, Gross C, Feger J, Boraud T, Bioulac B (1993). Reversal of rigidity and improvement in motor performance by subthalamic high-frequency stimulation in MPTP-treated monkeys. Eur J Neurosci 5: 382-389.

Benazzouz A, Piallat B, Pollak P, Benabid AL (1995). Responses of substantia nigra pars reticulata and globus pallidus complex to high frequency stimulation of the subthalamic nucleus in rats: electrophysiological data. Neurosci Lett 189: 77-80.

Berridge KC (2001). Reward learning: Reinforcement, incentives and expectations. In: Medin DL (ed). Psychology of Learning and Motivation: Advances in Research and Theory. Academic Press: New York, pp 223-278.

Breland K, Breland K (1961). The misbehavior of organisms. Am Psychol 16: 681-684.

Brown PL, Jenkins HM (1968). Auto-shaping of the pigeon's key-peck. J Exp Anal Behav 11: 1-8.

Charles PD, Van Blercom N, Krack P, Lee SL, Xie J, Besson G et al (2002). Predictors of effective bilateral subthalamic nucleus stimulation for PD. Neurology 59: 932-934.
Crombag HS, Badiani A, Maren S, Robinson TE (2000). The role of contextual vs discrete drug-associated cues in promoting the induction of psychomotor sensitization to intravenous amphetamine. Behav Brain Res 116: 1-22.

Crossman AR (1990). A hypothesis on the pathophysiological mechanisms that underlie levodopa- or dopamine agonistinduced dyskinesia in Parkinson's disease: implications for future strategies in treatment. Mov Disord 5: 100-108.

Davey GC, Cleland GG (1982). Topography of signal-centered behavior: effects of deprivation state and reinforcer type. J Exp Anal Behav 38: 291-304.

Davey GC, Cleland GG, Oakley DA, Jacobs JL (1984). The effect of early feeding experience on signal-directed response topography in the rat. Physiol Behav 32: 11-15.

Flagel SB, Watson SJ, Robinson TE, Akil H (2007). Individual differences in the propensity to approach signals vs goals promote different adaptations in the dopamine system of rats. Psychopharmacology (Berl) 191: 599-607.

Flagel SB, Watson SM, Akil H, Robinson TE (2008). Individual differences in the attribution of incentive salience to a rewardrelated cue: influence on cocaine sensitization. Behav Brain Res 186: $48-56$.

Florio T, Capozzo A, Cellini R, Pizzuti G, Staderini EM, Scarnati E (2001). Unilateral lesions of the pedunculopontine nucleus do not alleviate subthalamic nucleus-mediated anticipatory responding in a delayed sensorimotor task in the rat. Behav Brain Res 126: 93-103.

Funkiewiez A, Ardouin C, Krack P, Fraix V, Van Blercom N, Xie J et al (2003). Acute psychotropic effects of bilateral subthalamic nucleus stimulation and levodopa in Parkinson's disease. Mov Disord 18: 524-530.

Hearst E, Jenkins HM (1974). Sign tracking: the stimulusreinforcer relation and directed action. In: Monograph of the Psychonomic Society. Austin, Texas.

Houeto JL, Mesnage V, Mallet L, Pillon B, Gargiulo M, du Moncel ST et al (2002). Behavioural disorders, Parkinson's disease and subthalamic stimulation. J Neurol Neurosurg Psychiatry 72: 701-707.

Jenkins HM, Moore BR (1973). The form of the auto-shaped response with food or water reinforcers. J Exp Anal Behav 20: 163-181.

Limousin P, Krack P, Pollak P, Benazzouz A, Ardouin C, Hoffmann $\mathrm{D}$ et al (1998). Electrical stimulation of the subthalamic nucleus in advanced Parkinson's disease. N Engl J Med 339: 1105-1111.

Lozano AM, Dostrovsky J, Chen R, Ashby P (2002). Deep brain stimulation for Parkinson's disease: disrupting the disruption. Lancet Neurol 1: 225-231.

Lu C, Bharmal A, Suchowersky O (2006). Gambling and Parkinson disease. Arch Neurol 63: 298.

Newlin DB (1992). A comparison of drug conditioning and craving for alcohol and cocaine. Recent Dev Alcohol 10: 147-164.

Peterson GB, Ackilt JE, Frommer GP, Hearst ES (1972). Conditioned approach and contact behavior toward signals for food or brain-stimulation reinforcement. Science 177: 1009-1111.

Phillips JM, Brown VJ (1999). Reaction time performance following unilateral striatal dopamine depletion and lesions of the subthalamic nucleus in the rat. Eur J Neurosci 11: 1003-1010.

Robinson TE, Berridge KC (1993). The neural basis of drug craving: an incentive-sensitization theory of addiction. Brain Res Rev 18: 247-291.

Schupbach WM, Chastan N, Welter ML, Houeto JL, Mesnage V, Bonnet AM et al (2005). Stimulation of the subthalamic nucleus in Parkinson's disease: a 5-year follow-up. J Neurol Neurosurg Psychiatry 76: 1640-1644.

Smeding HM, Goudriaan AE, Foncke EM, Schuurman PR, Speelman JD, Schmand B (2007). Pathological gambling after bilateral subthalamic nucleus stimulation in Parkinson disease. J Neurol Neurosurg Psychiatry 78: 517-519. 
Tindell AJ, Berridge KC, Zhang J, Pecina S, Aldridge JW (2005). Ventral pallidal neurons code incentive motivation: amplification by mesolimbic sensitization and amphetamine. Eur $J$ Neurosci 22: 2617-2634.

Tomie A (1996). Locating reward cue at response manipulandum (CAM) induces symptoms of drug abuse. Neurosci Biobehav Rev 20: 505-535.

Uslaner JM, Acerbo MJ, Jones SA, Robinson TE (2006). The attribution of incentive salience to a stimulus that signals an intravenous injection of cocaine. Behav Brain Res 169: 320-324.

Uslaner JM, Robinson TE (2006). Subthalamic nucleus lesions increase impulsive action and decrease impulsive choice - mediation by enhanced incentive motivation? Eur J Neurosci 24: 2345-2354.

Uslaner JM, Yang P, Robinson TE (2005). Subthalamic nucleus lesions enhance the psychomotor-activating, incentive motivational, and neurobiological effects of cocaine. J Neurosci 25: 8407-8415.
Weeks JR (1972). Long-term intravenous infusion, In: Meyers RD (ed). Methods in Psychobiology. Academic Press, London. Vol 2, pp 155-168.

Welter ML, Houeto JL, Tezenas du Montcel S, Mesnage V, Bonnet AM, Pillon B et al (2002). Clinical predictive factors of subthalamic stimulation in Parkinson's disease. Brain 125: 575-583.

Williams DR, Williams H (1969). Automaintenance in the pigeon: sustained pecking despite contingent non-reinforcement. J Exp Anal Behav 12: 511-520.

Winstanley CA, Baunez C, Theobald DE, Robbins TW (2005). Lesions to the subthalamic nucleus decrease impulsive choice but impair autoshaping in rats: the importance of the basal ganglia in Pavlovian conditioning and impulse control. Eur J Neurosci 21: 3107-3116.

Wyvell CL, Berridge KC (2001). Incentive sensitization by previous amphetamine exposure: increased cue-triggered 'wanting' for sucrose reward. J Neurosci 21: 7831-7840. 